\title{
Melting temperature of diamond at ultrahigh pressure
}

\author{
J. H. Eggert ${ }^{1 \star}$, D. G. Hicks ${ }^{1}$, P. M. Celliers ${ }^{1}$, D. K. Bradley ${ }^{1}$, R. S. McWilliams ${ }^{1,2}$, R. Jeanloz ${ }^{2}$, J. E. Miller ${ }^{3}$, \\ T. R. Boehly ${ }^{3}$ and G. W. Collins ${ }^{1}$
}

Since Ross proposed that there might be 'diamonds in the sky' in 1981 (ref. 1), the idea of significant quantities of pure carbon existing in giant planets such as Uranus and Neptune has gained both experimental ${ }^{2}$ and theoretical ${ }^{3}$ support. It is now accepted that the high-pressure, high-temperature behaviour of carbon is essential to predicting the evolution and structure of such planets ${ }^{4}$. Still, one of the most defining of thermal properties for diamond, the melting temperature, has never been directly measured. This is perhaps understandable, given that diamond is thermodynamically unstable, converting to graphite before melting at ambient pressure, and tightly bonded, being the strongest bulk material known ${ }^{5,6}$. Shockcompression experiments on diamond reported here reveal the melting temperature of carbon at pressures of 0.6-1.1 TPa (6-11 Mbar), and show that crystalline diamond can be stable deep inside giant planets such as Uranus and Neptune ${ }^{1-4,7}$. The data indicate that diamond melts to a denser, metallic fluid-with the melting curve showing a negative Clapeyron slope-between 0.60 and $1.05 \mathrm{TPa}$, in good agreement with predictions of first-principles calculations ${ }^{8}$. Temperature data at still higher pressures suggest diamond melts to a complex fluid state, which dissociates at shock pressures between 1.1 and $2.5 \mathrm{TPa}$ (11-25 Mbar) as the temperatures increase above $50,000 \mathrm{~K}$.

As a result of the importance of high-pressure carbon in both planetary science and inertial confinement fusion (for which high-density carbon is a candidate ablator material for ignition target designs), there are many theoretical calculations of the high-pressure melting curve of diamond, with some predicting a maximum in temperature at $500 \mathrm{GPa}$ (5 Mbar; refs 8-11). Direct temperature measurements are challenging for both static and dynamic high-pressure experiments, so confirmation of meltcurve predictions have been only by inference, never by direct measurement. Under static conditions, equation-of-state data relevant to diamond melting do not extend to pressures much above $50 \mathrm{GPa}$, and are interpreted as indicating a positive Clapeyron slope, $(\partial T / \partial P)_{\text {melt }}>0$, for diamond up to $60 \mathrm{GPa}$ (refs 5, 6, 12, 13). Dynamic shock experiments on graphite, which is thought to convert to diamond under dynamic loading, suggest a positive melting slope to at least $140 \mathrm{GPa}$ (refs 14-16). Finally, recent shock experiments on diamond show an increase in density near $600 \mathrm{GPa}$, suggesting that shocked diamond might potentially melt with a negative melt curve near $600 \mathrm{GPa}$ (refs 17-20).

Here we report the first temperature measurements for shockcompressed diamond at conditions of $0.6-4 \mathrm{TPa}(6-40 \mathrm{Mbar})$ and $8,000-100,000 \mathrm{~K}$. These data reveal the melting curve for carbon up to $1.1 \mathrm{TPa}$ and a complex fluid state between 1.2 and $2.5 \mathrm{TPa}$.

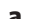

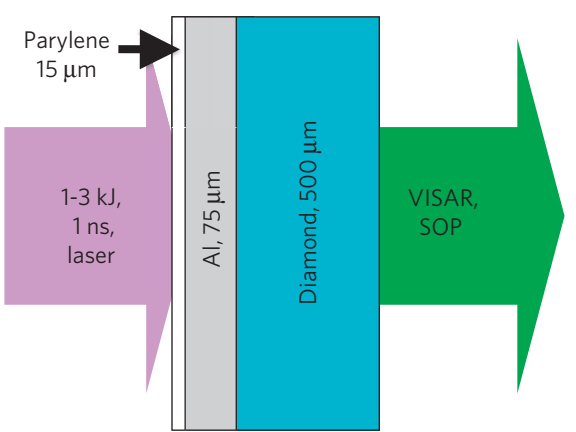

b
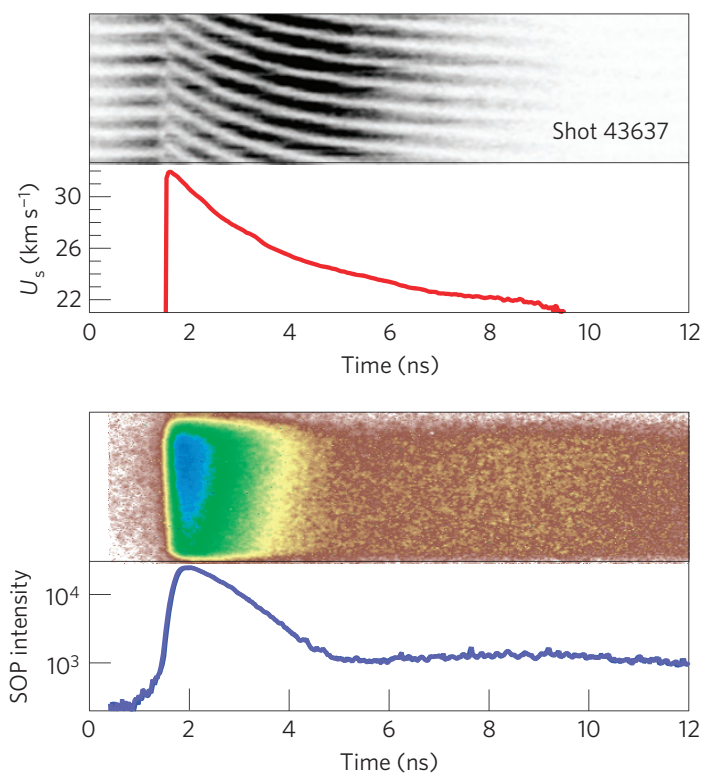

Figure 1 | Experimental configuration and data collected to determine the melting temperature of diamond. a, Diamond melt target. $\mathbf{b}$, Velocity interferometry (VISAR) data for polycrystalline diamond, showing raw fringe data and velocity $\left(U_{s}\right)$ lineout. The pressure falls continuously and smoothly with time as $U_{s}$ decreases. c, SOP data, showing raw data and intensity lineout. The SOP intensity plateaus and rises even as $U_{s}$ decreases smoothly.

Shock experiments traditionally study a steady-shock Hugoniot characterized by the shock velocity $U_{s}$, particle velocity $U_{\mathrm{p}}$, pressure $P$, specific volume $V,(=1 /$ density $)$ and internal energy $E$ of the shocked material. These five variables are related by the three Rankine-Hugoniot relations, so that the state of the sample under

\footnotetext{
${ }^{1}$ Lawrence Livermore National Laboratory, Livermore, California 94551, USA, ${ }^{2}$ University of California, Berkeley, California 94720, USA, ${ }^{3}$ University of Rochester, Rochester, New York 14623, USA. *e-mail: eggert1@llnl.gov.
} 


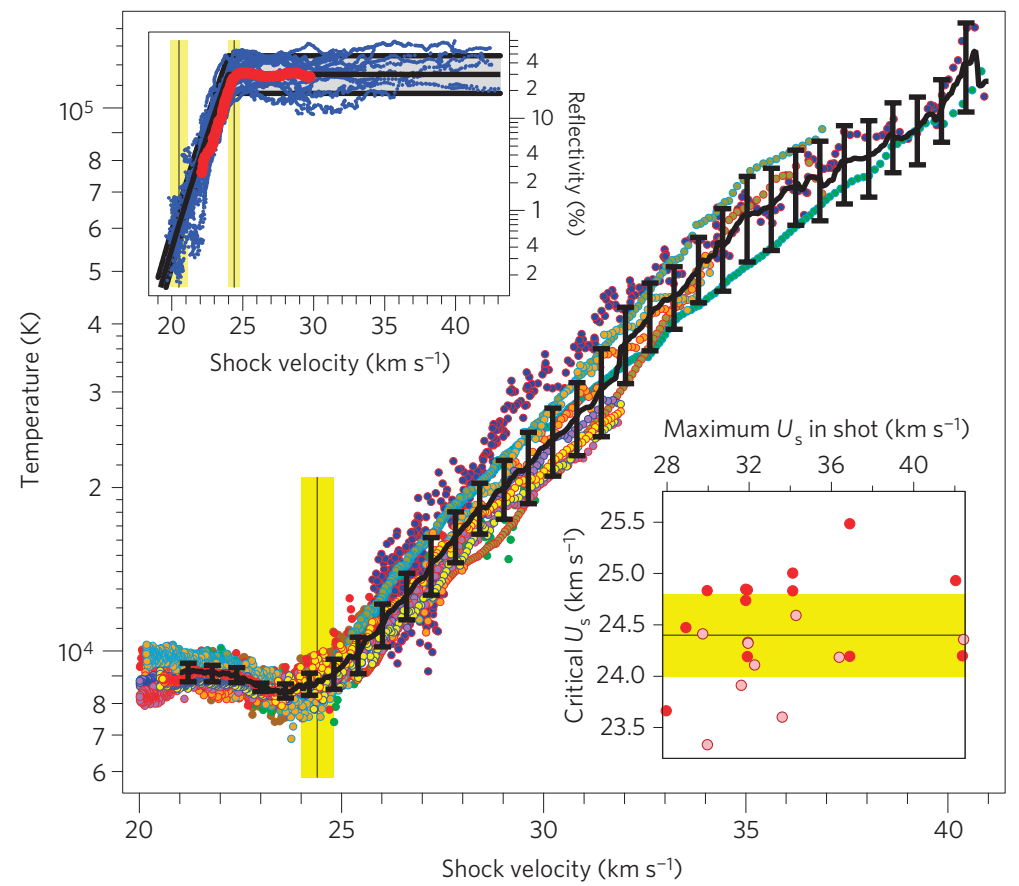

Figure 2 | Temperature versus shock velocity for all nine experiments carried out in the present study. As the shock-front pressure is decaying with time, the highest $T, P$ points correspond to early-time conditions. The upper left inset shows reflectivity measurements (blue) together with data from Bradley et al. ${ }^{7}$ (red). The black line is the averaged value. The lower right inset shows the fitted determination of $T-U_{\mathrm{s}}$ kinks (red) and $R_{\lambda=532}$ plateaux (pink) used to find the upper range of the mixed-phase region. The mean and standard deviation of these independent determinations are shown by yellow shaded lines throughout the figure, as is the shock velocity at $R_{\lambda}=532=0.5 \%$ in the upper left inset. The uncertainties shown in the main figure represent the weighted standard deviation of all measurements at each shock velocity as discussed in the Supplementary Information.

shock loading is determined by a single measurement if the $P-V-E$ Hugoniot has been previously determined. Our experiments use an unsupported shock for which the pressure decays with time, while at the shock front $P, V, E, U_{\mathrm{s}}$ and $U_{\mathrm{p}}$ still obey mass, momentum and energy conservation, and hence the RankineHugoniot relations ${ }^{21}$. Thus, the unsupported shock samples a continuum of Hugoniot states as the shock evolves through time and space. We measure the shock velocity $U_{s}$ in the present experiments, and use the Sesame Hugoniot ${ }^{22}$ that well represents the compilation of new Hugoniot experiments ${ }^{17-20}$. In addition, we measure the non-Rankine-Hugoniot variables $T$, and reflectivity, $R_{\lambda=532}$, of our sample at visible wavelengths $(\lambda=532 \mathrm{~nm})$.

We used the OMEGA laser at the University of Rochester to produce shocks in diamond targets by focusing $1 \mathrm{~ns}$ pulses with up to $3 \mathrm{~kJ}$ of $351 \mathrm{~nm}$ light to a flat spot $\sim 600 \mu \mathrm{m}$ in diameter (Fig. 1a). Both single-crystal, natural Type-1a diamond and polycrystalline, synthetic Type-2a diamond discs were used, the latter being formed by chemical vapour deposition and having an average grain size of about $130 \mu \mathrm{m}$ with little preferred orientation. Any differences due to sample type were well within our experimental uncertainties. Under ambient conditions, the density $\left(\rho_{0}\right)$ and refractive index $(n)$ of the diamond were determined to be $\rho_{0}=3.51( \pm 0.02) \mathrm{g} \mathrm{cm}^{-3}$ and $n=2.42( \pm 0.02)$ at $\lambda=532 \mathrm{~nm}$.

For most of its transit through the sample, the decaying shock front is reflecting and the instantaneous $U_{\mathrm{s}}$ and $R_{\lambda=532}$ are measured as a function of time using a velocity interferometer (VISAR) (Fig. 1b). $R_{\lambda=532}$ is obtained by comparing the intensity of the VISAR signal from the shock front in diamond with the pre-shock signal from an aluminium reference. Simultaneously, the absolute spectral radiance at two wavelengths, $I(\lambda)$ with $\lambda=650$ and $450 \mathrm{~nm}$, is measured as a function of time using a streaked optical pyrometer ${ }^{23}$ (SOP) and, in combination with $R_{\lambda=532}$, is used to determine $T$ (Fig. 1c; ref. 24) as discussed in the Supplementary Information.
When the unsupported shock enters the diamond at $1.8 \mathrm{~ns}, I(\lambda)$ (and $T$ ) rises quickly to a peak value (Fig. 1c). These early-time high- $P$ and $U_{\mathrm{s}}$ data correspond to the highest $T$ data at the far right in Fig. 2. Between $\sim 2$ and $5 \mathrm{~ns}, I(\lambda)$ decreases, as expected, owing to the decay of the shock pressure versus time (Fig. 1c). However, between 6 and $10 \mathrm{~ns}, I(\lambda)$ actually increases while $U_{s}$ and $P$ continue to decay. This increase of $I(\lambda)$ was observed in every experiment at $U_{s}^{\text {crit }}=24.6( \pm 0.4) \mathrm{km} \mathrm{s}^{-1}$ (Fig. 2). Similarly, $R_{\lambda=532}$ has a saturated value of $30( \pm 10) \%$ at the initially high shock $P$ and $U_{s}$, and then begins to decrease with decreasing $U_{\mathrm{s}}$ at $U_{s}^{\text {crit }}=24.3( \pm 0.3) \mathrm{km} \mathrm{s}^{-1}$ (Fig. 2, upper inset) until the shock front becomes opaque at $U_{s} \leq 20.5 \mathrm{~km} \mathrm{~s}^{-1}$. Combining the two results, we find a significant change in the diamond Hugoniot at a critical shock velocity of $24.4( \pm 0.4) \mathrm{km} \mathrm{s}^{-1}$ (Fig. 2, lower inset).

If there were no energy sink, the Hugoniot $T$ would decrease systematically as a function of $P$. Instead, we observe a plateau in shock $T$ between 0.6 and $1.1 \mathrm{TPa}$ (Fig. 3), which we interpret as owing to the latent heat of a phase transition. As only one plateau is observed up to very high $T$, we identify it with the transition from liquid to solid (corresponding to a two-phase region; ref. 25). Thus, we infer that diamond melts at about 9,000 K and between 0.60 and $1.05 \mathrm{TPa}$ along the Hugoniot. These conclusions are consistent with first-principles molecular-dynamics calculations of the melt curve (Fig. 3; refs 8, 11), the interpretation of the loss of free-surface reflectivity at $550 \mathrm{GPa}$ (ref. 7) and with recent measurements of small $U_{\mathrm{s}}-U_{\mathrm{p}}$ slope changes that also agree with the molecular-dynamics calculations ${ }^{8,19}$. Our experiments do not have the sensitivity to distinguish the proposed occurrence of the $\mathrm{BC} 8$ phase or diamond-BC8-liquid carbon triple point ${ }^{8,9,21}$.

Reflectivity data for shock-compressed diamond suggest that the fluid phase of carbon is conducting at $P$ and $T$ above $1 \mathrm{TPa}$ and $10,000 \mathrm{~K}$ (Fig. 2), in good agreement with first-principles calculations, which predict metallization of carbon in the fluid phase at comparable conditions ${ }^{8,26}$. $R_{\lambda=532}$ increases with $P$, from 


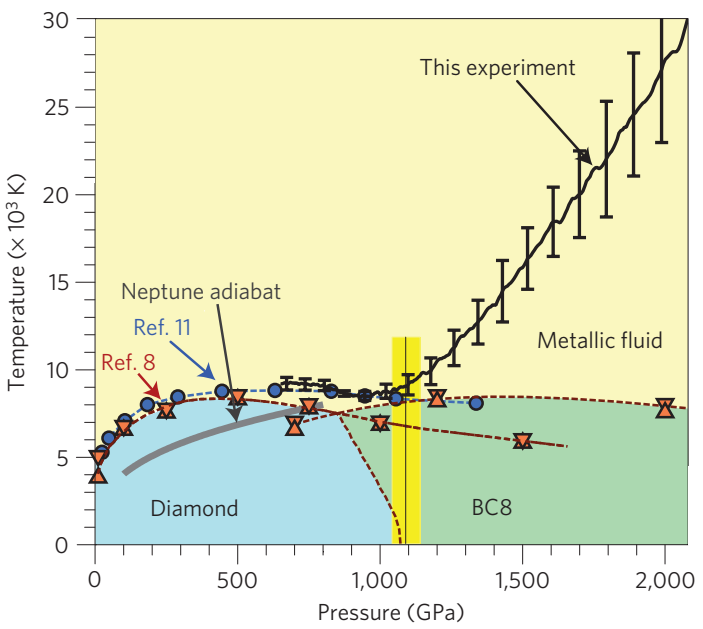

Figure 3 | Temperature versus pressure data compared with simulations. The black line denotes our new experimental shock temperature data. The blue circles and blue dashed line represent the melt curve from density functional theory calculations from Wang et al. that include diamond and liquid phases (ref. 11). The brown triangles that bracket the phase transitions and the brown dashed lines represent similar calculations by Correa et al. that include the diamond, BC8 and fluid phases (ref. 8). Our measurements are unable to identify multiple solid phases-be it diamond, $\mathrm{BC} 8$ or both-as predicted by the theory. The graphite phase is omitted for clarity. The uncertainties represent the weighted standard deviation of all measurements as discussed in the Supplementary Information.

below our detection threshold of $0.5 \%$ at $P<0.6 \mathrm{TPa}$ to $30 \%$ for $P>1.1 \mathrm{TPa}$. Applying a Drude model, these data give optical depths of $\sim 400 \mathrm{~nm}$ near $0.6 \mathrm{TPa}$, falling to $\sim 10 \mathrm{~nm}$ above $1.1 \mathrm{TPa}$, as discussed in the Supplementary Information. As atoms undergo more than 15 oscillations and the pressure decay is small during shock transit through an optical depth, the Hugoniot state is expected to be in local thermodynamic equilibrium. Although the transition from mixed-phase to fully solid carbon was not directly determined in the present experiments, both the reflectivity saturation in the pure fluid phase and the theoretical finding that the fluid is metallic ${ }^{8}$ suggest that the onset of melting be identified with the onset of reflectivity. The average $U_{\mathrm{s}}$ at $R_{\lambda}=532=0.5 \%$ (our detection limit) is $U_{S_{R=0.5 \%}}=20.5 \pm 0.6 \mathrm{~km} \mathrm{~s}^{-1}(0.60 \pm 0.06 \mathrm{TPa}$ and $9,200 \pm 800 \mathrm{~K}$ ).

Interpreting the diamond Hugoniot as following the melting curve from 0.6 to $1.05 \mathrm{TPa}$ allows us to estimate the Clapeyron slope, $\mathrm{d} T_{\mathrm{m}} / \mathrm{d} P=\Delta V_{\mathrm{m}} / \Delta S_{\mathrm{m}}=-2.6( \pm 0.6) \mathrm{K} \mathrm{GPa}^{-1}$ for the melting curve over this range. The entropy change, $\Delta S_{\mathrm{m}}$, is positive on melting, which implies that the volume change on melting is negative. A negative Clapeyron slope over this pressure range is in good agreement with the predictions of theory (Fig. 3), and a decrease in volume as the Hugoniot crosses the melting temperature of diamond is also supported by recent shock-compression experiments ${ }^{17-20}$.

Using our Hugoniot temperature measurements, we can extract the specific heat ${ }^{27}$, as discussed in the Supplementary Information. Just before melting, the sample is at a temperature much higher than the Debye temperature of diamond $(\sim 2,200 \mathrm{~K})$, so the specific heat should be close to the Dulong-Petit limit of $3 N k_{\mathrm{B}}$ ( $k_{\mathrm{B}}$ is Boltzmann's constant). In the fluid, the specific heat shows a broad peak with $C_{V} \approx 4.6 k_{\mathrm{B}}$ between 10,000 and $30,000 \mathrm{~K}$ (Fig. 4 ), implying an excess energy sink. At higher temperature, $C_{V}$ eventually approaches $3 N k_{\mathrm{B}}$ at about 70,000 K after first dropping below the Dulong-Petit limit. We consider it unlikely that this peak in $C_{V}$ is due to a change in ionization, because the reflectivity remains constant at about $30 \%$ over this entire $P-T$ range (Fig. 2, upper inset). High values of specific heat are similarly observed just above the melt temperatures

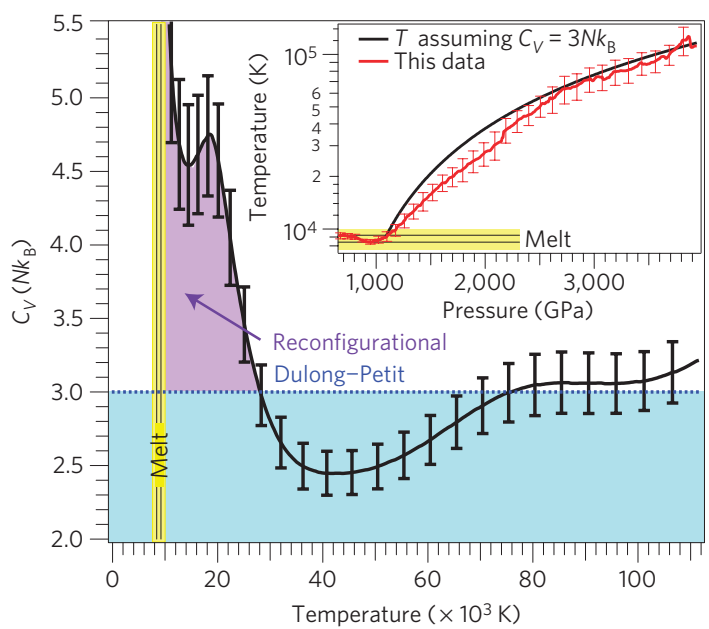

Figure 4 | Specific heat versus temperature showing the Dulong-Petit limit, $C_{V}=3 N_{B}$, which should hold for the solid below the melting temperature, and the peak attributed to atomic reconfiguration above the melting temperature. The uncertainties represent the standard deviation of results of a variety of assumptions including variations in the shock Hugoniot and the Gruneissen parameter discussed in the Supplementary Information. The inset compares temperature versus pressure from our measurements (red line) with the values calculated assuming a constant specific heat (black line). The temperatures for a hypothetical fluid obeying the Dulong-Petit limit are well above our uncertainties for pressures of 1.2-2.4 TPa. The temperature uncertainties are identical to those in Figs 2 and 3.

of quartz, fused silica, many alkali halides, silicon and germanium, and have been interpreted in terms of dissociation of a short-range ordered fluid. The high specific heat of $\mathrm{Si}$ and $\mathrm{Ge}$ is correlated with an anomalous liquid structure factor, and there is an explicit relationship between the structure factor and specific heat for a liquid described by pairwise interactions (equation 4.3.24; ref. 28). Thus, the observed peak in $C_{V}$ is probably due to a reconfiguration of atomic packing, from a partially bonded complex fluid to an atomic fluid above $60,000 \mathrm{~K}$. (References are presented in the Supplementary Information.)

Finally, estimates of the carbon mass fraction in Neptune and Uranus range from 11 to $17 \%$ in the $P-T$ region where $\mathrm{CH}_{4}$ has been observed to dissociate ${ }^{1-3,29}$. It is likely that much of this atomic carbon has gravitationally settled into a carbon-rich phase near the core of these planets ${ }^{1,3}$. Along with our melting temperatures, the model adiabat shown in Fig. 3 suggests that pure carbon could be solid at all depths, although it is possible that a hotter deep interior may exist, dynamically isolated from the outermost atmosphere by a stably stratified, non-convecting region within Uranus and Neptune $e^{4,30}$. In such warmer conditions, pure carbon would exist in the liquid metallic state, settling out of the mantle to form a fluid outer core that helps sustain the planetary magnetic field. In either case, the high sound velocity of a diamond-rich layer at intermediate depths could influence planetary normal modes that may be observable in the future.

Received 7 April 2009; accepted 25 September 2009; published online 8 November 2009

\section{References}

1. Ross, M. The ice layer in Uranus and Neptune-diamonds in the sky? Nature 292, 435-436 (1981).

2. Benedetti, L. R. et al. Dissociation of $\mathrm{CH}_{4}$ at high pressures and temperatures: Diamond formation in giant planet interiors? Science 286, 100-102 (1999).

3. Ancilotto, F., Chiarotti, G. L., Scandolo, S. \& Tosatti, E. Dissociation of methane into hydrocarbons at extreme (planetary) pressure and temperature. Science 275, 1288-1290 (1997). 
4. Guillot, T. The interiors of giant planets: Models and outstanding questions. Annu. Rev. Earth Planet. Sci. 33, 493-530 (2005).

5. Bundy, F. P. Melting of graphite at very high pressure. J. Chem. Phys. 38, 618-630 (1963).

6. Togaya, M. in Science and Technology of New Diamond (eds Saitro, S., Fukunaga, O. \& Yoshikawa, M.) 369-373 (KTK Scientific Publishers, 1990).

7. Bradley, D. K. et al. Shock compressing diamond to a conducting fluid. Phys. Rev. Lett. 93, 195506 (2004).

8. Correa, A. A., Bonev, S. A. \& Galli, G. Carbon under extreme conditions: Phase boundaries and electronic properties from first-principles theory. Proc. Natl Acad. Sci. USA 103, 1204-1208 (2006).

9. Grumbach, M. P. \& Martin, R. M. Phase diagram of carbon at high pressures and temperatures. Phys. Rev. B 54, 15730-15741 (1996).

10. Wu, C. J., Glosli, J. N., Galli, G. \& Ree, F. H. Liquid-liquid phase transition in elemental carbon: A first-principles investigation. Phys. Rev. Lett. 89, 135701 (2002).

11. Wang, X.-F., Scandolo, S. \& Car, R. Carbon phase diagram from ab initio molecular dynamics. Phys. Rev. Lett. 95, 185701 (2005).

12. Weathers, M. S. \& Bassett, W. A. Melting of carbon at 50-300 kbar. Phys. Chem. Minerals 15, 105-112 (1987).

13. Bundy, F. P. Direct conversion of graphite to diamond in static pressure apparatus. J. Chem. Phys. 38, 631-643 (1963).

14. Gust, W. H. Phase transition and shock-compression parameters to $120 \mathrm{GPa}$ for three types of graphite and for amorphous carbon. Phys. Rev. B 22, 4744-4756 (1980).

15. Fried, L. E. \& Howard, W. M. Explicit Gibbs free energy equation of state applied to the carbon phase diagram. Phys. Rev. B 61, 8734-8743 (2000).

16. Shaner, F. W., Brown, J. M., Swenson, C. A. \& McQueen, R. G. Sound velocity of carbon at high pressures. J. Phys. (Paris), Colloq. 45, C8-235-237 (1984).

17. Hicks, D. G. et al. High precision measurements of the diamond Hugoniot in and above the melt region. Phys. Rev. B 78, 174102 (2008).

18. Nagao, H. et al. Hugoniot measurement of diamond under laser shock compression up to 2 TPa. Phys. Plasmas 13, 052705 (2006).

19. Knudson, M. D., Desjarlais, M. P. \& Dolan, D. H. Shock-wave exploration of the high-pressure phases of carbon. Science 322, 1822-1825 (2008).

20. Brygoo, S. et al. Laser-shock compression of diamond and evidence of a negative slope melting curve. Nature Mater. 6, 274-277 (2007).
21. Hicks, D. G. et al. Dissociation of liquid silica at high pressures and temperatures. Phys. Rev. Lett. 97, 025502 (2006).

22. Lyon, S. P. \& Johnson, J. D. Diamond Sesame Table. Report No. LA-UR-92-3407 (Los Alamos National Laboratory, 1992).

23. Miller, J. E. et al. Streaked optical pyrometer system for laser-driven shock-wave experiments on OMEGA. Rev. Sci. Instrum. 78, 034903 (2006).

24. Collins, G. W. et al. Temperature measurements of shock compressed liquid deuterium up to 230 GPa. Phys. Rev. Lett. 87, 165504 (2001).

25. Kormer, S. B. Optical study of the characteristics of shock-compressed condensed dielectrics. Sov. Phys. Usp. 11, 229-254 (1968).

26. Scandolo, S., Chiarotti, G. L. \& Tosatti, E. SC4: A metallic phase of carbon at terapascal pressures. Phys. Rev. B 53, 5051-5054 (1996).

27. Keeler, R. N. \& Royce, E. B. in Physics of High Energy, Density (eds Caldirola, P. \& Knoepfel, H.) 88 (Academic, 1971).

28. Goodstein, D. L. States of Matter (Dover, 1985).

29. Hubbard, W. B. et al. Interior structure of Neptune: Comparison with Uranus. Science 253, 648-651 (1991).

30. Stanley, S. \& Bloxham, J. Convective-region geometry as the cause of Uranus' and Neptune's unusual magnetic fields. Nature 428, 151-153 (2004).

\section{Acknowledgements}

We thank W. Unites for sample support. This work was carried out under the auspices of the US Department of Energy by Lawrence Livermore National Laboratory under Contract DE-AC52-07NA27344.

\section{Author contributions}

J.H.E., D.G.H., P.M.C., D.K.B. and T.R.B. designed and carried out the experiments. J.H.E., D.G.H., P.M.C. and R.S.M. carried out the analysis; J.E.M. and T.R.B. calibrated the SOP. R.J. and G.W.C. administered the experiment and J.H.E., R.J. and G.W.C. wrote the manuscript.

\section{Additional information}

The authors declare no competing financial interests. Supplementary information accompanies this paper on www.nature.com/naturephysics. Reprints and permissions information is available online at http://npg.nature.com/reprintsandpermissions. Correspondence and requests for materials should be addressed to J.H.E. 